\title{
Variational Methods, Bounds and Size Effects for Composites with Highly Conducting Interface
}

\author{
Robert Lipton \\ Department of Mathematical Sciences \\ Worcester Polytechnic Institute \\ 100 Institute Road \\ Worcester, MA 01609*
}

May 3,1996

\begin{abstract}
New variational principles are developed for the effective conductivity tensor for anisotropic two-phase electric conductors. Here the interface between phases is assumed to be highly conducting. Extra geometric information is encoded into the principles through the solution operators of simpler transport problems. These operators can be expressed as gradients of simple layer potentials with densities supported on phase interfaces or in terms or simple Dirichlet problems inside each phase region.

New upper bounds on the effective conductivity are found that depend upon component volume fractions, a surface energy tensor and a scale-free matrix of parameters. This matrix corresponds to the effective conductivity tensor associated with the same geometry but with perfectly conducting inclusions. New lower bounds are given in terms of two-point correlation functions, component volume fractions, and interfacial geometric parameters. Both upper and lower bounds are found to be optimal for certain choices of interfacial parameters.

For isotropic polydisperse suspensions of spheres we are able to estimate the effective conductivity based on measured values of the size distribution of the spheres. Conversely, we are able to characterize the size distribution of the spherical inclusions based on measured values of the effective conductivity.
\end{abstract}

\section{Introduction}

We consider two-phase electric conductors with phases separated by a highly conducting interface. Such an interface may be thought of as the limiting case of electrical transport

*Research supported by AFOSR grant F49620-96-1-0055 and NSF grant DMS-9205158. 
between phases separated by a thin highly conducting interphase layer. The highly conducting interface is characterized by a discontinuous current field across the interface. The jump in current normal to the interface produces a charge density on the interface. The associated electric potential is continuous across the interface and is coupled to the interfacial charge density through a Poisson equation supported on the interface, see equation (2.3). Existence and uniqueness of the solutions to the field equations have been established by Pham Huy and Sanchez-Palencia (1974).

We investigate the effective electrical conductivity in the context of a periodic arrangement of conductors. Aside from the requirement of periodicity, the distribution of the phases within the period call can be arbitrary. To fix ideas, we assume that the two phases consist of isotropic conductors, with conductivities specified by $\sigma_{2}>\sigma_{1}$ in the proportions $\theta_{1}$ and $\theta_{2}$, respectively. The highly conducting interface is characterized by a constant, scalar, "tangential conductivity" denoted by $\alpha$. We treat both two and three dimensional composite geometries. Here, a two dimensional composite geometry corresponds to parallel cylinders of conductivity $\sigma_{1}$ and $\sigma_{2}$. For this case we investigate the effective conductivity in the plane transverse to the generators of the cylinders.

In this paper we provide new variational principles from which new bounds on effective properties are obtained through simple choices of trial fields, (see Sections 3,5 and 6.) The extension of the variational principles to the multi-phase case is straight forward and is discussed in Section 9.

This work is motivated by the idea that variational principles containing extra geometric information provide tighter bounds than those obtainable from Dirichlet or Thompson variational principles for any given class of trial fields. The variational principles introduced here incorporate geometric information through the solution operators of simpler comparison problems. These operators admit an explicit representation either in terms of gradients of simple layer potentials supported on the two-phase interface, projection operators on the space $L^{2}(Q)^{d}$, or are associated with simple Dirichlet problems in each phase. (see Section 3.)

New bounds and variational principles for two-phase conductors with contact resistance have been derived using this approach by Lipton and Vernescu in (1996). Moreover this approach has been successful in the context of two-phase elastic composites with imperfect bonding at the two-phase interface. See Lipton and Vernescu (1995).

Substitution of simple trial fields into the upper variational principle (3.12) yields a new upper bound on the effective conductivity that depends upon component volume fractions, a surface energy tensor and a scale free matrix of parameters. For particulate composites, this matrix corresponds to the effective conductivity tensor associated with the same geometry but with perfectly conducting inclusions. The surface energy tensor appearing in the upper bound (5.9) is identical (up to sign) with the surface energy tensor introduced by Chandrasekhar (1965) for the stability analysis of rotating liquid drops held together by 
surface tension. For particulate composites, the new lower bound (6.10) is given in terms of the two point correlation function, component volume fractions, and geometric parameters of the interface phase.

When the composite geometry is isotropic the bounds simplify. We consider the behavior of the bounds in the specific surface area "s" and a geometric parameter " $m$ " of the interface. Here " $\mathrm{m}$ " represents the total polar moment of inertia of the interface. To fix ideas we note that for a polydisperse suspension of " $\mathrm{N}$ " spheres with radii $a_{1}, a_{2}, \ldots, a_{N}$ with prescribed volume fraction $\theta_{1}$, the geometric parameter of the interface " $\mathrm{m}$ " is given by $\theta_{1}\langle a\rangle / 2$ and the total specific interfacial surface area is $s=3 \theta_{1}\left\langle a^{-1}\right\rangle$. Here the averages of a quantity $q_{i}, i=1,2, \ldots, N$ over all spheres is given by $\langle q\rangle=\theta_{1} \sum_{i=1}^{N}\left(\left|Y_{i}\right| / \theta_{1}\right) q_{i}$, where $\left|Y_{i}\right|$ is the volume occupied by the $i^{\text {th }}$ sphere.

We find that in the limit $s \rightarrow \infty$, that the upper bound is linked to the effective properties of suspensions of perfectly conducting inclusions, (Section 7 ). Indeed, it follows from Lipton (1995), that the asymptotic behavior of the bounds is consistent with the effective properties for a large class of composites in the high surface area limit. Physically this follows from the behavior of the effective property under a rescaling of the geometry as observed in Lipton (1995) (Theorem 4.2). This topic is pursued in Section 7.

In Sections 5 and 6 , the bounds are shown to be monotonic with respect to the geometric parameters of the interface. This property is used in Section 7 to identify a parameter $P_{c r}^{d}=(d-1) \alpha\left(\sigma_{2}-\sigma_{1}\right)^{-1}(d=2,3)$. This parameter measures the relative importance of the tangential conductivity with respect to the contrast between bulk conductivities. Here $d$ gives the dimension of the composite. Physically speaking this parameter picks out the length scale for which effects of the interface balance the mismatch between the conductivities of each phase. This is clearly seen for isotropic monodisperse suspensions of spheres of conductivity $\sigma_{1}$ in a matrix of conductivity $\sigma_{2}$. Indeed, for isotropic monodisperse suspensions of spheres of radius " $P_{c r}^{d}$ ", the effective conductivity $\sigma^{e}$ is precisely the matrix conductivity $\sigma_{2}$, see Theorem 7.1. This result has been observed earlier by Torquato and Rintoul (1995) and by Lipton (1995). The method of Torquato and Rintoul considers classical Thompson and Dirichlet variational principles for three phase statistically isotropic composites. Here the two bulk phases are $\sigma_{2}$ and $\sigma_{1}$ and the third phase is a thin highly conducting interphase layer of conductivity $\sigma_{i}$ and width $l$. By means of an elegant choice of trial fields, the upper and lower bounds are found to agree at critical radius $P_{c r}^{3}$, in the finite limit of $l \sigma_{i}$, when $l$ tends to zero and $\sigma_{i}$ tends to infinity. On the other hand, the approach presented in Lipton (1995) calculates the fields inside the composite to find that the electric field is uniform throughout the composite for suspensions of spheres (disks) at critical radius $P_{c r}^{d} d=2,3$. From this it follows that the inclusions are hidden even when the monodisperse distribution of spheres is statistically anisotropic, see Lipton (1995). This observation is not possible using the methods of Torquato and Rintoul (1995). 
In this paper we extend the known results to include size effects for isotropic polydisperse suspensions of spheres and for inclusions of any shape and distribution see Theorem 7.1, 7.2, and 7.3. For polydisperse suspension of spheres at fixed volume fraction, it is shown that the effective conductivity always lies above that of the matrix provided that the mean radius of the polydisperse suspension lies below $P_{c r}^{d}$. When the harmonic mean of the radii are above $P_{c r}^{d}$ then the effective conductivity always lies below the matrix (see Theorem 7.2.) More generally, for isotropic suspensions of particles of any shape and distribution we find that the effective conductivity lies below that of the matrix when the specific surface area to particle volume fraction lies below $d\left(P_{c r}^{d}\right)^{-1}, d=2,3$ (see Theorem 7.3.) These theorems are applied to address the role of surface energy when designing energy minimizing arrangements of inclusions (see Theorem 7.4.) Here, we fix the volume fraction and find a necessary condition on the size distribution for the isotropic polydisperse suspension with minimal effective conductivity.

We remark that in two dimensions a reciprocal relation was obtained in Lipton (1995) relating the effective conductivity function to the effective conductivity of a composite with interfacial contact resistance. This reciprocal relation together with new bounds on the effective conductivity tensor for composites with interfacial contact resistance introduced in Lipton and Vernescu (1996) were applied to obtain bounds on the effective conductivity tensor for composites with highly conducting interface, see Lipton (1995). The two dimensional versions of the bounds obtained in this paper naturally agree those given in Lipton (1995) (see Sections 5 and 6.)

The monotonicity of the bounds can be used to solve inverse problems. In Section 8, we show how to apply measured values of the effective conductivity to characterize the size distribution for isotropic, polydisperse suspensions of spheres.

Lastly, we note that although we have used the terminology of electrical conductivity, our results apply equally to the contexts of thermal conductivity, magnetic permeability, and diffusivity.

\section{Effective Conductivity for Composites with Highly Con- ducting Interface and a New Thompson Variational Prin- ciple}

We consider a unit cube $Q$ filled with two isotropic conductors with conductivities specified by $\sigma_{1}$ and $\sigma_{2}$. In what follows we make no assumption on the distribution of the conductors within the interior of the domain. One can think of the cube as representing a (possibly very complicated) period cell for a composite material. Decomposing the electric potential 
into a periodic fluctuation $\tilde{\varphi}$ and a linear part $\mathbf{E} \cdot x$ the average electric field inside $Q$ is:

$$
\mathbf{E}=\int_{\partial Q}(\tilde{\varphi}+\mathbf{E} \cdot x) \mathbf{n} d s .
$$

Here $\partial Q$ is the boundary of the cube and $\mathbf{n}$ is the outer normal to the boundary. To fix ideas we assume that the two-phase boundary is sufficiently smooth (i.e., a twice differentiable surface). The fluctuating part of the potential is continuous across phase interfaces and satisfies:

$$
\Delta \tilde{\varphi}=0 \quad \text { inside each phase }
$$

and:

$$
\sigma_{1}(\nabla \tilde{\varphi}+\mathbf{E})_{1} \cdot \mathbf{n}-\sigma_{2}(\nabla \tilde{\varphi}+\mathbf{E})_{2} \cdot \mathbf{n}=\alpha \Delta(\tilde{\varphi}+\mathbf{E} \cdot x)
$$

on the phase boundary $\Gamma$. Subscripts 1 and 2 denote the side of the interface where field quantities are evaluated. Here $\mathbf{n}$ is the unit normal pointing into phase 2 , and $\Delta$ is the Laplace-Beltrami operator on $\Gamma$ defined by

$$
\Delta(\tilde{\varphi}+\mathbf{E} \cdot x)=\delta_{i} \delta_{i}(\tilde{\varphi}+\mathbf{E} \cdot x)
$$

where $\delta$ is the tangential gradient of $\tilde{\varphi}+\mathbf{E} \cdot x$ on $\Gamma$, i.e.,

$$
\delta_{i}(\tilde{\varphi}+\mathbf{E} \cdot x)=\partial_{x_{i}}(\tilde{\varphi}+\mathbf{E} \cdot x)-(\mathbf{n} \cdot(\nabla \tilde{\varphi}+\mathbf{E})) \mathbf{n}_{i} .
$$

We observe from (2.3) that the current suffers a discontinuity at the two-phase interface. The jump in current provides a surface charge density which drives the Poisson equation (2.3) on the two-phase interface.

Denoting the local conductivity by $\sigma(x)$ the (possibly anisotropic) effective conductivity tensor $\sigma^{e}$ of the mixture as measured by an outside observer is defined as:

$$
\sigma^{e} \mathbf{E}=\int_{\partial Q} \sigma(x)(\nabla \tilde{\varphi}+\mathbf{E}) \cdot \mathbf{n} x d S .
$$

When $\alpha=0$ we recover the well known case of perfectly bonded composites. The limit $\alpha=\infty$ corresponds to a composite filled with perfectly conducting inclusions.

Integration by parts and application of (2.2), (2.3) and the natural boundary condition for the current yields:

$$
\sigma^{e} \mathbf{E} \cdot \mathbf{E}=\int_{Q} \sigma(x)|\nabla \tilde{\varphi}+\mathbf{E}|^{2} d x+\alpha \int_{\Gamma}|\delta(\tilde{\varphi}+\mathbf{E} \cdot x)|^{2} d s .
$$

Physically equation (2.7) is a relation between the total energy dissipation rate inside the heterogeneous conductor and the energy dissipated in a homogeneous effective conductor.

One easily verifies the Dirichlet variational principle for the effective conductivity:

$$
\sigma^{e} \mathbf{E} \cdot \mathbf{E}=\min _{\varphi \in V} \int_{Q} \sigma(x)|\nabla \varphi+\mathbf{E}|^{2} d x+\alpha \int_{\Gamma}|\delta(\varphi+\mathbf{E} \cdot x)|^{2} d s .
$$


where the space of trial fields is given by:

$$
V=\left\{\varphi \in W^{1,2}(Q) \mid \varphi-Q \text { periodic }\right\}
$$

We represent a jump in a quantity " $q$ " across the two-phase boundary by $[q]=q_{1}-q_{2}$. The unit cell admits the partition $Q=Q_{1} \cup Q_{2} \cup \Gamma$, where $Q_{1}$ and $Q_{2}$ are the parts of $Q$ occupied by conductor one and two respectively. We consider particulate composites for which the particles do not intersect the walls of the period cell. We make this assumption for convenience only and to keep the exposition focused. (What follows can be applied to any periodic two-phase geometry.) We denote the surface of the $i^{\text {th }}$ particle by $\Gamma_{i}$ and write $\Gamma=\bigcup_{i} \Gamma_{i}$. We introduce the space of trial fields $\mathcal{C}$ defined by

$$
\begin{aligned}
& \mathcal{C}=\left\{\tilde{p} \text { in } L^{2}(Q)^{d}\right. \text {, where : } \\
& \text { i) } \operatorname{div} \tilde{p}=0 \text { in } Q_{1} \cup Q_{2}, \int_{\partial Q} \tilde{p} \cdot \mathbf{n} x d s=0, \\
& \text { ii) } \int_{\Gamma_{i}}[\tilde{p} \cdot \mathbf{n}] d s=0 \text { and } \\
& \text { iii) } \tilde{p} \cdot \mathbf{n} \text { anti-periodic on } Q \cdot\}
\end{aligned}
$$

Next we introduce the solution $g$ in $H^{1}(\Gamma)$ of the Poisson equation on the interface given by

$$
\Delta g=[\tilde{p} \cdot \mathbf{n}] \text { on } \Gamma_{i}
$$

We observe that $g$ is determined uniquely up to a constant on each particle surface for $\tilde{p}$ satisfying the solvability condition given by ii).

We state the new Thompson variational principle for composites with highly conducting interface:

Theorem 2.1 For all $\bar{p}$ in $\mathbb{R}^{d}$ one has

$$
\sigma^{e^{-1}} \bar{p} \cdot \bar{p}=\min _{\tilde{p} \in \mathcal{C}}\left\{\int_{Q} \sigma^{-1}(x)|\tilde{p}+\bar{p}|^{2}+\alpha^{-1} \int_{\Gamma}|\delta g|^{2} d s\right\} .
$$

Proof: One could proceed applying the methods of duality theory given in Ekeland and Temam (1976) to the Dirichlet-like variational principle (2.8). However, we proceed with a hands on approach. Starting with (2.8) we take the convex dual in each of the terms on the right hand side to obtain:

$$
\begin{aligned}
\sigma^{e} \mathbf{E} \cdot \mathbf{E}= & \min _{\varphi \in V} \max _{p \in L^{2}(Q)^{d}} \max _{v \in L^{2}(\Gamma)}\left\{2 \int_{Q} p \cdot(\nabla \varphi+\mathbf{E})-\int_{Q} \sigma^{-1} p \cdot p+\right. \\
& \left.+2 \int_{\Gamma} v \cdot \delta(\varphi+\mathbf{E} \cdot x)-\alpha^{-1} \int_{\Gamma}|v|^{2}\right\} .
\end{aligned}
$$


Exchanging max and min implies that the trial fields $p$ and $v$ must be chosen such that

$$
\min _{\varphi \in V}\left\{\int_{Q} p \cdot \nabla \varphi+\int_{\Gamma} v \cdot \delta \varphi d s\right\}>-\infty
$$

Integration by parts reveals that (2.14) is equivalent to the constraints $\operatorname{div} p=0$ in $Q_{1} \cup Q_{2}$, $[p \cdot \mathbf{n}]=\delta_{i} v_{i}+d(v \cdot \mathbf{n}) \mathcal{H}$ on $\Gamma$, and $p \cdot \mathbf{n}$ is anti-periodic. Here the mean curvature of $\Gamma$ is written as $\mathcal{H}=-d^{-1} \operatorname{div} \mathbf{n}$, and for any $v$ in $L^{2}(\Gamma)$ the tangential derivitave $\delta_{i} v_{i}$ is defined in the distributional sense.

Substitution of these identities into (2.13) together with the identity

$$
\int_{Q_{1} \cup Q_{2}} \operatorname{div}(\mathbf{E} \cdot x p)-\int_{\partial Q}(\mathbf{E} \cdot x) p \cdot \mathbf{n} d s=\int_{\Gamma}(\mathbf{E} \cdot x)[p \cdot \mathbf{n}] d s
$$

gives

$$
\sigma^{e} \mathbf{E} \cdot \mathbf{E} \geq \sup _{(p, v)}\left\{2 \int_{\partial Q} p \cdot \mathbf{n} x d s \cdot \mathbf{E}-\int_{Q} \sigma^{-1} p \cdot p-\int_{\Gamma} \alpha^{-1}|v|^{2} d s\right\} .
$$

Setting $\bar{p}=\int_{\partial Q}(p \cdot \mathbf{n}) x d s$ and $\tilde{p}=\bar{p}-p$, it follows from (2.6) that $\bar{p}=\sigma^{e} \mathbf{E}$ or $\sigma^{e^{-1}} \bar{p}=\mathbf{E}$ and so,

$$
\sigma^{e^{-1}} \bar{p} \cdot \bar{p} \leq \min _{(\tilde{p}, v)}\left\{\int_{Q} \sigma^{-1}(\tilde{p}+\bar{p}) \cdot(\tilde{p}+\bar{p})+\alpha^{-1} \int_{\Gamma}|v|^{2} d s\right\} .
$$

For the choice $\tilde{p}+\bar{p}=\sigma(x)(\nabla \tilde{\varphi}+\mathbf{E}), \mathbf{E}=\sigma^{e^{-1}} \bar{p}$, and $v=\alpha \delta(\varphi+\mathbf{E} \cdot x)$ one satisfies the constraints implied by (2.14) and obtains equality in (2.16). Last we note that the optimal choice of $v$ in (2.16) is of the form $\delta g$ where $g$ satisfies (2.11) and the Theorem follows.

\section{New Variational Principles}

In this Section, we introduce new variational principles for the effective conductivity tensor. Before giving the first variational principle we introduce a homogeneous comparison material with conductivity $\gamma>\sigma_{2}$ and formulate three auxiliary conductivity problems. For any constant $\mathbf{c}$ in $\mathbb{R}^{d}(d=2,3)$ we introduce the potential $\psi^{c}$ in $V$ which solves:

$$
\begin{aligned}
\psi^{\mathbf{c}}+\mathbf{c} \cdot x & =0 & & \text { on } \Gamma, \\
\Delta \psi^{\mathbf{c}} & =0 & & \text { in } Q_{1} \cup Q_{2} .
\end{aligned}
$$

We introduce the space $H\left(\operatorname{div}, Q_{1} \cup Q_{2}\right)$ of square integrable vector fields $\eta$ for which div $\eta$ lies in $L^{2}\left(Q_{1} \cup Q_{2}\right)$. We denote by $\mathcal{P}$ the space:

$$
\mathcal{P}=\left\{\eta \text { in } H\left(\text { div }, Q_{1} \cup Q_{2}\right) \text { such that } \eta \cdot \mathbf{n} \text { is anti-periodic. }\right\}
$$


Then for $\eta$ in $\mathcal{P}$ and $q$ in $H^{1}(\Gamma)$ we have the potentials $\psi^{q}$ and $\psi^{\eta}$ that are solutions of:

$$
\begin{aligned}
\psi^{q}=q, & \text { on } \Gamma \\
\Delta \psi^{q}=0, & \text { on } Q_{1} \cup Q_{2}, \text { and } \\
\psi^{\eta}=0, & \text { on } \Gamma, \\
\Delta \psi^{\eta}=\operatorname{div} \eta & \text { on } Q_{1} \cup Q_{2} .
\end{aligned}
$$

We introduce the linear operators $M, R, P$ defined by:

$$
M(\mathbf{c})=\nabla \psi^{\mathbf{c}} \text { on } Q_{1} \cup Q_{2}, \quad R(q)=\nabla \psi^{q} \text { on } Q_{1} \cup Q_{2},
$$

and

$$
P(\eta)=\nabla \psi^{\eta} \quad Q_{1} \cup Q_{2}
$$

Next, we define the constant $d \times d$ tensor $\sigma_{\infty}$ by:

$$
\sigma_{\infty} \mathbf{c}=\int_{\partial Q}\left(\nabla \psi^{\mathbf{c}}+\mathbf{c}\right) \cdot \mathbf{n} x d s,
$$

for any $\mathbf{c}$ in $\mathbb{R}^{d}, d=2,3$, and introduce the surface energy tensor given by:

$$
\mathcal{G}=-d \int_{\Gamma}(x \otimes n) \mathcal{H} d s
$$

where $\mathcal{H}$ is the mean curvature of the interface. One can show that $\sigma_{\infty}^{-1}$ exists, this is established in the Appendix. We introduce the space of trial fields $U$ given by $U=\mathcal{P} \times H^{1}(\Gamma)$ and state the new upper variational principle given by:

Theorem 3.1 For any vector $\bar{p}$ in $\mathbb{R}^{d}$ one has

$$
\begin{aligned}
& \left(\sigma^{e^{-1}}-\gamma^{-1}\right) \bar{p} \cdot \bar{p}+\frac{\alpha}{\gamma^{2}} \mathcal{G} \bar{p} \cdot \bar{p}= \\
& =\max _{(\eta, q) \in U}\left[2 \int_{Q} \bar{p} \cdot \eta d x+2 \frac{\alpha}{\gamma} \int_{\Gamma} \delta q \cdot(I-n \otimes n) \bar{p} d s-\right. \\
& -\int_{Q}\left(\sigma^{-1}-\gamma^{-1}\right) \eta \cdot \eta-\alpha \int_{\Gamma}|\delta q|^{2} d s- \\
& \left.-\gamma \int_{Q}\left|M\left(\sigma_{\infty}^{-1}(\bar{\eta}+\bar{q})\right)+R(q)+P(\eta)+\sigma_{\infty}^{-1}(\bar{\eta}+\bar{q})-\eta\right|^{2} d x\right] \text {, }
\end{aligned}
$$

where

$$
\bar{\eta}=\int_{\partial Q}\left(\eta-\nabla \psi^{\eta}\right) \cdot \boldsymbol{n} x d s, \bar{q}=-\int_{\partial Q} \nabla \psi^{q} \cdot \boldsymbol{n} x d s .
$$


We note here that the tensor " $\mathcal{G}$ " appearing in Theorem 3.1 is (up to sign) identical to the surface energy tensor introduced in Chandrasekhar [1] for the stability analysis of a rotating liquid drop held together by surface tension.

Before introducing the lower variational principle we select an isotropic comparison material with conductivity $\gamma<\sigma_{1}$, and introduce the linear operators $N$ and $S$. Here, for any $\eta$ in $L^{2}(Q)^{d}$, we have $N \eta$ in $L^{2}(Q)^{d}$, where

$$
N \eta=-\frac{1}{\gamma} \sum_{k \neq 0} e^{2 \pi i k \cdot x} \frac{\kappa \hat{\eta}(\kappa) \cdot \kappa}{|\kappa|^{2}}
$$

and for any vector field $v$ defined on $\Gamma$ with components $v_{i}$ in $H^{1}(\Gamma)$ we have $S v$ in $L^{2}(Q)^{d}$ where

$$
S v=\left(\frac{-1}{2 \pi i}\right) \gamma^{-1} \sum_{k \neq 0} e^{2 \pi i k \cdot x} \frac{\kappa}{|\kappa|^{2}} \int_{\Gamma} e^{-2 \pi i \kappa \cdot y} T v d s .
$$

Here $T v$ is given by:

$$
T v=\delta_{i} v_{i}+d(v \cdot \mathbf{n}) \mathcal{H}
$$

and

$$
\hat{\eta}(\kappa)=\int_{Q} e^{-2 \pi i \kappa \cdot y} \eta(\psi) d y
$$

We denote the set of vector fields defined on $\Gamma$ with components in $H^{1}(\Gamma)$ by $W$ and introduce the space $\mathcal{L}$ of trial fields, $\mathcal{L}=L^{2}(Q)^{d} \times W$, and state the new lower variational principle:

Theorem 3.2 For any $E$ in $\mathbb{R}^{d}$,

$$
\begin{gathered}
\left(\sigma^{e}-\gamma\right) \boldsymbol{E} \cdot \boldsymbol{E}=\max _{(p, v) \text { in } \mathcal{L}}\left[2 \int_{Q} p \cdot \boldsymbol{E} d x+2 \int_{\Gamma} v \cdot(I-\boldsymbol{n} \otimes \boldsymbol{n}) \boldsymbol{E} d s\right. \\
\int_{Q}(\sigma-\gamma)^{-1}|p|^{2} d x-\alpha^{-1} \int_{\Gamma}|v|^{2} d s- \\
\left.-\gamma \int_{Q}|N p+S v|^{2} d x\right] .
\end{gathered}
$$

We remark that the operator $N$ is proportional to the projection of $L^{2}(Q)^{d}$ onto the space of gradients of potential functions in $V$, (c.f. (2.9)). This operator is well known and forms the basis for the Hashin Shtrikman bounds for anisotropic conductors with perfectly bonded interfaces given by Kohn and Milton (1988). The $S$ operator given by (3.15) is the gradient in $Q_{1} \cup Q_{2}$ of the simple layer potential with density $T v$ on the two phase interface. 


\section{Derivation of the Variational Principles}

To derive the upper variational principle given by Theorem 3.1 we start with the Thompson variational principle given by Theorem 2.1. We choose $\gamma>\sigma^{2}$ and add and subtract a reference energy $\gamma|\tilde{p}+\bar{p}|^{2}$ to the right-hand side of (2.11) to obtain:

$$
\begin{aligned}
\left(\sigma^{e^{-1}}-\gamma^{-1}\right) \bar{p} \cdot \bar{p} & =\min _{\tilde{p} \in \mathcal{C}}\left[\int_{Q}\left(\sigma(x)^{-1}-\gamma^{-1}\right)|\tilde{p}+\bar{p}|^{2} d x+\gamma^{-1} \int_{Q}|\tilde{p}|^{2} d x\right. \\
& \left.+2 \gamma^{-1} \int_{Q} \tilde{p} \cdot \bar{p} d x+\alpha^{-1} \int_{\Gamma}|\delta g|^{2} d s\right] .
\end{aligned}
$$

Since $\tilde{p}$ lies in $\mathcal{C}$ and $\Delta g=[\tilde{p} \cdot \mathbf{n}]$, integration by parts gives:

$$
2 \gamma^{-1} \int_{Q} \tilde{p} \cdot \bar{p} d x=2 \gamma^{-1} \int_{\Gamma}[\tilde{p} \cdot n] x \cdot \bar{p} d s=2 \gamma^{-1} \int_{\Gamma} \Delta g x \cdot \bar{p} d s .
$$

Integration by parts on $\Gamma$ yields:

$$
2 \gamma^{-1} \int_{Q} \tilde{p} \cdot \bar{p} d x=2 \gamma^{-1} \int_{\Gamma} \delta g \cdot(I-\mathbf{n} \otimes \mathbf{n}) \bar{p} d s .
$$

Next we apply (4.3) and complete the square in (4.1) to obtain

$$
\begin{aligned}
\left(\sigma^{e^{-1}}-\gamma^{-1}\right) \bar{p} \cdot \bar{p} & +\frac{\alpha}{\gamma^{2}} \mathcal{G} \bar{p} \cdot \bar{p}=\min _{\tilde{p} \in \mathcal{C}}\left[\int_{Q}\left(\sigma(x)^{-1}-\gamma^{-1}\right)|\tilde{p}+\bar{p}|^{2} d x+\right. \\
& \left.+\gamma^{-1} \int_{Q}|\tilde{p}|^{2} d x+\alpha^{-1} \int_{\Gamma}\left|\delta g-\frac{\alpha}{\gamma}(I-\mathbf{n} \otimes \mathbf{n}) \bar{p}\right|^{2} d s\right]
\end{aligned}
$$

Here

$$
\mathcal{G}=\int_{\Gamma}(I-\mathbf{n} \otimes \mathbf{n}) d s=-d \int_{\Gamma} x \otimes \mathbf{n} \mathcal{H} d s
$$

is a surface energy tensor which up to sign agrees with the surface energy tensor introduced in [1]. Equation (4.5) follows from integrating by parts on the surface $\Gamma$.

Introducing the bulk and surface polarizations $\eta$ in $\mathcal{P}$, (cf. 3.3), and $q$ in $H^{1}(\Gamma)$ we have the elementary estimates.

$$
\int_{Q}\left(\sigma^{-1}-\gamma^{-1}\right)|\tilde{p}+\bar{p}|^{2} d x \geq 2 \int_{Q}(\bar{p}+\tilde{p}) \cdot \eta-\int_{Q}\left(\sigma^{-1}-\gamma^{-1}\right)^{-1} \eta \cdot \eta
$$

and

$$
\begin{aligned}
\alpha^{-1} \int_{\Gamma}\left|\delta g-\frac{\alpha}{\gamma}(I-\mathbf{n} \otimes \mathbf{n}) \bar{p}\right|^{2} d s & \geq 2 \int_{\Gamma} \delta q \cdot\left(\delta g-\frac{\alpha}{\gamma}(I-\mathbf{n} \otimes \mathbf{n}) \bar{p}\right) d s \\
& -\alpha \int_{\Gamma}|\delta q|^{2} d s .
\end{aligned}
$$


Applying (4.6) and (4.7) to (4.4) we obtain

$$
\left(\sigma^{e^{-1}}-\gamma^{-1}+\frac{\alpha}{\gamma^{2}} \mathcal{G}\right) \bar{p} \cdot \bar{p} \geq \min _{\tilde{p} \in \mathcal{C}} \overline{\mathcal{L}}(\tilde{p}, \eta, q)=\overline{\mathcal{L}}(\dot{\tilde{p}}, \eta, q)
$$

where $\overline{\mathcal{L}}$ is defined by

$$
\begin{aligned}
\overline{\mathcal{L}}(\tilde{p}, \eta, q) & =2 \int_{Q} \bar{p} \cdot \eta d x-2 \frac{\alpha}{\gamma} \int_{\Gamma} \delta q \cdot(I-\mathbf{n} \otimes \mathbf{n}) \bar{p} d s-\int_{Q}\left(\sigma^{-1}-\gamma^{-1}\right)^{-1} \eta \cdot \eta d x \\
& -\alpha \int_{\Gamma}|\delta q|^{2}+2 \int_{Q} \tilde{p} \cdot \eta d x+2 \int_{\Gamma} \delta q \cdot \delta g d s+\gamma^{-1} \int_{Q}|\tilde{p}|^{2} d x
\end{aligned}
$$

and $\stackrel{*}{p}$ is the minimizer of

$$
\overline{\mathcal{J}}=\min _{\tilde{p} \in \mathcal{C}}\left\{2 \int_{Q} \tilde{p} \cdot \eta d x+2 \int_{\Gamma} \delta q \cdot \delta g d s+\gamma^{-1} \int_{Q}|\tilde{p}|^{2} d x\right\} .
$$

Since $\Delta g=[\tilde{p} \cdot \mathbf{n}]$, integration by parts in the middle term of (4.10) shows that the minimum value $\overline{\mathcal{J}}$ is given by

$$
\overline{\mathcal{J}}=\min _{\tilde{p}}\left\{2 \int_{Q} \tilde{p} \cdot \eta d x-2 \int_{\Gamma} q[\tilde{p} \cdot n] d s+\gamma^{-1} \int_{Q}|\tilde{p}|^{2} d x\right\}
$$

for all $\tilde{p}$ satisfying (2.10) (i) and (iii). Taking the first variation, one finds that the minimizer $\stackrel{*}{p}$ is given by

$$
\stackrel{\tilde{\tilde{p}}}{=} \gamma(\nabla \ddot{\psi}+c-\eta),
$$

where $\psi$ is in $V$, (cf. (2.9)), and $\mathbf{c}$ is a constant vector. The function $\psi$ and $\mathbf{c}$ are solutions of the system:

$$
\begin{aligned}
\psi+\mathbf{c} \cdot x=q & \text { on } \Gamma, \\
\Delta \psi=\nabla \cdot \eta & \text { in } Q_{1} \cup Q_{2}, \text { and } \\
\int_{\partial Q}(\nabla \psi+\mathbf{c}-\eta) \cdot \mathbf{n} x d s=0 . &
\end{aligned}
$$

In order to solve for $\psi$ and $c$ simultaneously in (4.13)-(4.15) we make use of the linearity inherent in the problem and form the three auxiliary problems $(3.1)-(3.2),(3.4)-(3.5)$, and (3.6)-(3.7). One readily sees that the choice

$$
\mathbf{c}=\sigma_{\infty}^{-1}(\bar{\eta}+\bar{q})
$$

and $\psi=\psi^{e}+\psi^{q}+\psi^{\eta}$ is the solution for the system (4.13)-(4.15). Recalling the definitions of the operators $M, R$, and $P$ we have the inequality:

$$
\left(\sigma^{e^{-1}}-\gamma^{-1}+\frac{\alpha}{\gamma^{2}} \mathcal{G}\right) \bar{p} \cdot \bar{p} \geq \max _{(\eta, q) \in U} \overline{\mathcal{L}}(\tilde{\tilde{p}}, \eta, q)=
$$




$$
\begin{aligned}
= & \max _{(\eta, q) \in U}\left[2 \int_{Q} \bar{p} \cdot \eta d x+2 \frac{\alpha}{\gamma} \int_{\Gamma} \delta q \cdot(I-\mathbf{n} \otimes \mathbf{n}) \bar{p} d s-\right. \\
& -\int_{Q}\left(\sigma^{-1}-\gamma^{-1}\right) \eta \cdot \eta-\alpha \int_{\Gamma}|\delta q|^{2} d s- \\
& \left.-\gamma \int_{Q}\left|M\left(\sigma_{\infty}^{-1}(\bar{\eta}+\bar{q})\right)+R(q)+P(\eta)+\sigma_{\infty}^{-1}(\bar{\eta}+\bar{q})-\eta\right|^{2} d x\right] .
\end{aligned}
$$

For the choice of bulk and surface polarizations, consistent with the actual current and potential fields in the composite, i.e.,

$$
\eta=\left(\sigma^{-1}-\gamma^{-1}\right)(\tilde{p}+\bar{p}), \quad q=\tilde{\varphi}+\mathbf{E} \cdot x-\gamma^{-1} \bar{p} \cdot x,
$$

where $\mathbf{E}=\sigma^{e^{-1}} \bar{p}$, we find that $\mathbf{c}=\left(\sigma^{e^{-1}}-\gamma^{-1}\right) \bar{p}$ and one observes that (4.17) holds with equality. This establishes the upper variational principle.

Next we establish Theorem 3.2 for the choice of isotropic comparison material $\gamma<\sigma_{1}$. Starting with the Dirichlet-like variational principle given by (2.8), we add and subtract the reference energy $\gamma|\nabla \varphi+\mathbf{E}|^{2}$ to obtain

$$
\begin{gathered}
\left(\sigma^{e}-\gamma\right) \mathbf{E} \cdot \mathbf{E}=\min _{\varphi \in V}\left[\int_{Q}(\sigma(x)-\gamma)|\nabla \varphi+\mathbf{E}|^{2} d x+\int_{Q} \gamma|\nabla \varphi|^{2} d x+\right. \\
\left.+\alpha \int_{\Gamma}|\delta(\varphi+\mathbf{E} \cdot x)|^{2} d s\right]
\end{gathered}
$$

One has the elementary estimates,

$$
\int_{Q}(\sigma(x)-\gamma)|\nabla \varphi+\mathbf{E}|^{2} d x \geq 2 \int_{Q} p \cdot(\nabla \varphi+\mathbf{E}) d x-\int_{Q}(\sigma(x)-\gamma)^{-1}|p|^{2} d x,
$$

and

$$
\alpha \int_{\Gamma}|\delta(\varphi+\mathbf{E} \cdot x)|^{2} d s \geq 2 \int_{\Gamma} v \cdot \delta(\varphi+\mathbf{E} \cdot x) d s-\alpha^{-1} \int_{\Gamma}|v|^{2} d s,
$$

for all polarizations $(p, v)$ in $L^{2}(Q) \times L^{2}(\Gamma)$. Application of the estimates to (4.19) yield

$$
\left(\sigma^{e}-\gamma\right) \mathbf{E} \cdot \mathbf{E} \geq \min _{\varphi \in V} \underline{\mathcal{L}}(p, v, \varphi)=\underline{\mathcal{L}}\left(p, v, \varphi^{*}\right)
$$

where $\underline{\mathcal{L}}$ is defined by

$$
\begin{aligned}
\underline{L}(p, v, \varphi) & =2 \int_{Q} p \cdot \mathbf{E} d x+2 \int_{\Gamma} v \cdot \delta(\mathbf{E} \cdot x) d s- \\
& -\int_{Q}(\sigma-\gamma)^{-1}|p|^{2} d x-\alpha^{-1} \int_{\Gamma}|v|^{2} d s+ \\
& +2 \int_{Q} p \cdot \nabla \varphi+2 \int_{\Gamma} v \cdot \delta \varphi d s+\gamma \int_{Q}|\nabla \varphi|^{2},
\end{aligned}
$$


and $\varphi^{*}$ is the minimizer of

$$
\underline{\mathcal{J}}=\min _{\varphi \in V}\left[2 \int_{Q} p \cdot \nabla \varphi+2 \int_{\Gamma} v \cdot \delta \varphi d s+\gamma \int_{Q}|\nabla \varphi|^{2}\right] .
$$

Taking the first variation one finds that the minimizer $\varphi^{*}$ is the solution of:

$$
\gamma \Delta \dot{\varphi}=-\operatorname{div} p \quad \text { in } Q_{1} \cup Q_{2}
$$

and

$$
[\gamma \nabla \ddot{\varphi}+p] \cdot \mathbf{n}=\delta_{i} v_{i}+d(v \cdot \mathbf{n}) \mathcal{H} \text { on } \Gamma .
$$

Since (4.25), (4.26) are linear we may write $\ddot{\varphi}=\varphi^{p}+\varphi^{v}$ where both $\varphi^{p}$ and $\varphi^{v}$ are elements of $V$ and

$$
\gamma \Delta \varphi^{p}=-\operatorname{div} p \text { in } Q_{1} \cup Q_{2},\left[\gamma \nabla \varphi^{p}+p\right] \cdot \mathbf{n}=0 \text { on } \Gamma,
$$

and

$$
\gamma \Delta \varphi^{v}=0 \text { in } Q_{1} \cup Q_{2},\left[\gamma \nabla \varphi^{v}\right] \cdot \mathbf{n}=\delta_{i} v_{i}+d(v \cdot \mathbf{n}) \mathcal{H} \text { on } \Gamma \text {. }
$$

Writing $N p=\nabla \varphi^{p}, S v=\nabla \varphi^{v}$ for $x$ in $Q_{1} \cup Q_{2}$ and observing that $\delta(\mathbf{E} \cdot x)=(I-\mathbf{n} \otimes n) \mathbf{E}$ on $\Gamma$ we have the inequality

$$
\begin{aligned}
\left(\sigma^{e}-\gamma\right) \mathbf{E} \cdot \mathbf{E} \geq \max _{(p, v)} \underline{\mathcal{L}}\left(p, v, \varphi^{*}\right)= \\
=\max _{(p, v)}\left[2 \int_{Q} p \cdot \mathbf{E} d x+2 \int_{\Gamma} v \cdot(I-\mathbf{n} \otimes \mathbf{n}) \mathbf{E} d s-\int_{Q}(\sigma-\gamma)^{-1}|p|^{2} d x-\right. \\
\left.-\alpha^{-1} \int_{\Gamma}|v|^{2} d s-\gamma \int_{Q}|N p+S v|^{2} d x\right] .
\end{aligned}
$$

In fact, equality in (4.29) is easily seen to hold when the polarizations $(p, v)$ are chosen to correspond with the actual fields in the composite, i.e.,

$$
p=(\sigma(x)-\gamma)(\nabla \tilde{\varphi}+\mathbf{E}), \quad v=\alpha \delta(\tilde{\varphi}+E \cdot x) .
$$

To finish the proof we show that the operators $N p=\nabla \varphi^{p}$ and $S v=\nabla \varphi^{v}$ are given by the explicit formulas (3.14) and (3.15) respectively. The explicit formula (3.14) for the operator $N$ follows immediately from solution of the comparison problem (4.27) using Fourier Series expansions. To obtain the representation of the $S$ operator we extend the density $T v$ (cf. 3.16) into $Q_{1}$ via the solution $\psi$ of the boundary value problem

$$
\begin{aligned}
\Delta \psi=0 & \text { in } Q_{1} \\
\partial_{n} \psi=T v & \text { on } \Gamma
\end{aligned}
$$

and

$$
\psi-Q \text { periodic on } \partial Q_{1} \cap \partial Q
$$


Introducing $\chi_{1}$, the characteristic function of $Q_{1}$, i.e., $\chi_{1}=1$ in $Q_{1}, \chi_{1}=0$ otherwise; it is evident that $\varphi^{v}$ is the unique solution (up to a constant) of:

$$
\gamma \Delta \varphi^{\nu}=\operatorname{div}\left(\chi_{1} \nabla \psi\right) \text { on } Q
$$

in the weak sense. Fourier expansion gives

$$
S v=\gamma^{-1} \sum_{\kappa \neq 0} e^{2 \pi i \kappa \cdot x} \frac{\kappa}{|\kappa|^{2}} \int_{Q_{1}} e^{-2 \pi i \kappa \cdot y} \kappa \cdot \nabla \psi d y .
$$

The desired representation for $S$ follows upon integration by parts in (4.35) and application of (4.32).

\section{$5 \quad$ Upper Bounds}

We apply Theorem 3.1 to obtain new upper bounds on the effective tensor. To fix ideas we consider anisotropic particulate composites with particles of conductivity $\sigma_{1}$ in a connected matrix of $\sigma_{2}$. We denote the region occupied by the $i^{\text {th }}$ particle by $Y_{i}$ and its boundary by $\partial Y_{i}$.

We choose trial polarizations of the form $\eta=\chi_{1} \mu, q=r \cdot x$ on each particle surface and set $\gamma=\sigma_{2}$ in (3.12). Here $\mu$ and $r$ are vectors in $\mathbb{R}^{d}$. The associated bound is given by:

\section{Theorem 5.1}

$$
\left(\sigma^{e^{-1}}-\sigma_{2}^{-1}+\alpha \sigma_{2}^{-2} \mathcal{G}\right) \bar{p} \cdot \bar{p} \geq \max _{\substack{\mu \in \mathbb{R}^{d} \\ r \in \mathbb{R}^{d}}}\{2 \bar{L}(\bar{p}, \mu, r)-\bar{Q}(\mu, r)\}
$$

where

$$
\bar{L}(\bar{p}, \mu, r)=2 \theta_{1} \bar{p} \cdot \mu-2 \alpha \sigma_{2}^{-2} \mathcal{G} r \cdot \bar{p}
$$

and

$$
\bar{Q}(\mu, r)=\theta_{1} \lambda|\mu|^{2}+\alpha \mathcal{G} r \cdot r+\sigma_{2}\left(\left(I-\dot{\sigma}_{\infty}^{-1}\right) r \cdot r-2 \theta_{1} r \cdot \mu+\theta_{1}|\mu|^{2}\right),
$$

here $\lambda=\left(\sigma_{1}^{-1}-\sigma_{2}^{-1}\right)^{-1}$, and $\tilde{\sigma}_{\infty}^{*}$ is the effective conductivity tensor of a composite with perfectly conducting particles having the same geometry as the original composite embedded in a matrix of unit conductivity.

Proof: We observe that (5.2) and the first two terms of (5.3) follow immediately upon substitution of the polarizations into (3.12). The last three terms in (5.3) follow from the solutions of the comparison problems. Indeed, for $\eta=\chi_{1} \mu$ and $q=r \cdot x$ on each particle one finds that $\psi^{\eta}=0$, and $\psi^{q}$ affine inside each particle i.e., $\psi^{q}=r \cdot x$ in $Y_{i}$. Moreover $\psi^{q}$ is the solution of:

$$
\Delta \psi^{q}=0 \quad \text { in } Q_{2}
$$


and

$$
\psi^{q}=r \cdot x \quad \text { on } \Gamma=\bigcup_{i} \partial Y_{i} .
$$

From the definition of $\sigma_{\infty}$ given by (3.10) it follows that

$$
\bar{q}=-\int_{\delta Q} \nabla \psi^{q} \cdot n \times d s=\left(\sigma_{\infty}-I\right) r .
$$

Since $\bar{\eta}=0$ equation (4.16) yields:

$$
\mathbf{c}=\sigma_{\infty}^{-1} \bar{q}=\left(I-\sigma_{\infty}^{-1}\right) r
$$

It follows after a lengthy but straightforward application of (5.4)-(5.7) together with (3.1), (3.2) that:

$$
\begin{gathered}
\int_{Q}\left|M\left(\sigma_{\infty}^{-1}(\bar{\eta}+\bar{q})\right)+R(q)+P(\eta)+\sigma_{\infty}^{-1}(\bar{\eta}+\bar{q})-\eta\right|^{2} d x= \\
=\left(I-\sigma_{\infty}^{-1}\right) r \cdot r-2 \theta_{1} r \cdot \mu+\theta_{1}|\mu|^{2} .
\end{gathered}
$$

Last, for particulate geometries one readily observes that $\psi^{\mathbf{c}}=-\mathbf{c} \cdot x$ inside each particle and that the tensor $\sigma_{\infty}$ reduces to $\stackrel{*}{\infty}_{\infty}$ stated in Theorem 5.1, see Appendix. This completes the proof.

Optimizing over all constants $\mu$ and $r$ gives the following explicit upper bound for anisotropic particulate composites.

Theorem 5.2 For any $\bar{p}$ in $\mathbb{R}^{d}$ one has the inequality:

$$
\begin{aligned}
& \sigma_{e}^{-1} \bar{p} \cdot \bar{p} \geq\left(\sigma_{2}^{-1}-\alpha \sigma_{2}^{-2} \mathcal{G}\right) \bar{p} \cdot \bar{p}+ \\
& +\left(\begin{array}{cc}
\theta_{1}\left(\lambda+\sigma_{2}\right) I & -\sigma_{2} \theta_{1} I \\
-\sigma_{2} \theta_{1} I & \alpha \mathcal{G}+\sigma_{2}\left(I-\sigma_{\infty}^{*}\right)
\end{array}\right)^{-1}\left(\begin{array}{c}
\theta_{1} \bar{p} \\
\frac{-\alpha}{\sigma_{2}^{2}} \mathcal{G} \bar{p}
\end{array}\right) \cdot\left(\begin{array}{c}
\theta_{1} \bar{p} \\
\frac{-\alpha}{\sigma_{2}^{2}} \mathcal{G} \bar{p}
\end{array}\right)
\end{aligned}
$$

For isotropic composites the surface energy tensor $\mathcal{G}$ reduces to $\mathcal{G}=\left(\frac{d-1}{d}\right) s I$ and the upper bound becomes:

\section{Corollary 5.3}

$$
\sigma^{e} \leq U B\left(s \alpha, \sigma_{\infty}^{*}\right)=\frac{\sigma_{2}\left(\theta_{1} Z+\sigma_{2}\left(1-\dot{\sigma}_{\infty}^{-1}\right)\right)}{\sigma_{\infty}^{*} \theta_{1} Z+\sigma_{2}\left(1-\tilde{\sigma}_{\infty}^{-1}\right)}
$$

where $Z$ is the characteristic combination

$$
Z=\left(\frac{d-1}{d}\right) \frac{s \alpha}{\theta_{1}}-\left(\sigma_{2}-\sigma_{1}\right)
$$


It is easily checked that this bound is monotone increasing with respect to the specific interfacial surface area $s$ and tangential conductivity $\alpha$. We note that for $d=2$ this bound is precisely the upper bound on the effective conductivity obtained in Lipton (1995) using reciprocal relations together with lower bounds on effective properties for composites with interfacial contact resistance.

For isotropic polydisperse suspensions of spheres one easily calculates that

$$
s=d \theta_{1}\left\langle a^{-1}\right\rangle
$$

where \langle\rangle is the average reciprocal radius taken over all spheres in the suspension. For monodisperse suspensions we observe that $s=3 \theta_{1} a$ and for $a=P_{c r}^{d}$ the upper bound becomes

$$
U B\left(d \theta_{1}\left(P_{c r}^{d}\right)^{-1} \alpha, \stackrel{*}{\sigma}_{\infty}\right)=\sigma_{2} .
$$

More generally, for any isotropic suspension one has

$$
U B\left(s \alpha, \stackrel{\sigma}{\infty}_{\infty}\right)=\sigma_{2}
$$

when $s=d \theta_{1}\left(P_{c r}^{d}\right)^{-1}$.

Lastly, we observe that the upper bound is monotonic increasing in the parameter $\sigma_{\infty}^{*}$. From the monotonicity properties we have

$$
\sigma^{e} \leq U B\left(\alpha s, \stackrel{\sigma}{\infty}_{\infty}\right)<U B\left(\infty, \stackrel{\sigma}{\infty}_{\infty}\right)=\sigma_{2} \sigma_{\infty}^{*}
$$

and

$$
\sigma^{e} \leq U B\left(\alpha s, \dot{\sigma}_{\infty}\right)<U B(\alpha s, \infty)=\left(\frac{d-1}{d}\right) s \alpha+\theta_{1} \sigma_{1}+\theta_{2} \sigma_{2} .
$$

The right-most upper bound in (5.15) is the effective conductivity of the same suspension but with perfectly conducting particles. The right-most bound in (5.16) corresponds with an arithmetic mean type upper bound in terms of the tangential conductivity and the two bulk conductives.

\section{Lower Bounds}

In this Section we make specific choices of trial fields in (3.17) to obtain new lower bounds for three-dimensional anisotropic particulate suspensions of conductor 1 in a matrix of conductor 2. We start with a choice of trials for which the integrals appearing in the variational principle are easy to compute for any particle shape. The resulting bounds are general and hold for all suspensions of particles independently of the shape and location of the particles, see (6.13). We also present similar bounds for anisotropic two-dimensional composites, see (6.14). If one knows the shape and size distribution of the particles then one can tailor the trial fields to the inclusion shape. To fix ideas we consider an isotropic 
polydisperse suspension of spheres. Here we choose surface polarizations to be scaled affine functions. This choice is motivated by the fact that affine functions are eigenfunctions of the Laplacian on the sphere, c.f., Kobayashi and Nomizu (1969).

To start, we introduce the vector $y^{i}=x-r^{i}$ where $x$ lies on the surface $\partial Y^{i}$ of the $i^{\text {th }}$ particle and $r^{i}$ is the center of mass inside the particle. We choose $p=\chi_{2} \mu$ where $\chi_{2}$ is the indicator function of the matrix and $\mu$ is a vector in $\mathbb{R}^{3}$. For $r$ in $\mathbb{R}^{3}$ we introduce the polarization $v=r_{j} z^{j}$ on $\partial Y^{i}$, were $z^{j}=n \times h^{j}$ such that curl $h^{j}=e^{j}$, and $e^{j}$ is the $j^{\text {th }}$ unit vector for an orthogonal system. On the surface of the $i^{\text {th }}$ particle $h^{1}=y_{2}^{i} e^{3}, h^{2}=y_{3}^{i} e^{1}$, and $h^{3}=y^{1} e^{2}$.

We set $\gamma=\sigma_{1}$ and substitute these trial fields into (3.17) to find:

\section{Theorem 6.1}

$$
\left(\sigma^{e}-\gamma\right) \boldsymbol{E} \cdot \boldsymbol{E} \geq \max _{\substack{\mu \text { in } \mathbb{R}^{3} \\ r \text { in } \mathbb{R}^{3}}}[2 \underline{L}(\mu, r, \boldsymbol{E})-\underline{Q}(\mu, r)]
$$

where

$$
\begin{aligned}
\underline{L}(\mu, r, \boldsymbol{E}) & =2 \theta_{2} \mu \cdot \boldsymbol{E}+2 \theta_{1} r \cdot \boldsymbol{E}, \\
\underline{Q}(\mu, r) & =\theta_{2}\left(\sigma_{2}-\sigma_{1}\right)^{-1}|\mu|^{2}+\alpha^{-1} \mathbb{Z} r \cdot r-\sigma_{1}^{-1} \mathbb{T}(r-\mu) \cdot(r-\mu) .
\end{aligned}
$$

Here $\mathbb{Z}$ is a symmetric matrix of interfacial geometric parameters with components

$$
\mathbb{Z}^{k \ell}=\sum_{i} \int_{\partial Y^{i}}\left(h^{k} \cdot h^{\ell}\right)-\left(n \cdot h^{k}\right)\left(n \cdot h^{\ell}\right) d s,
$$

and

$$
\mathbb{T}=\int_{Q} \chi_{2} \mathbb{P} \chi_{2} d x,
$$

where $\mathbb{P}$ is the projection of $L^{2}(Q)^{3}$ onto the space of gradients of periodic potential fields in the space $V$ given by (2.9).

Proof: Equation (6.2) and the first two terms of (6.3) follow directly from substitution of the trials into the first four terms of (3.17). The last term of $(6.3)$ follows from the identity

$$
\sigma_{1} \int_{Q}|N p+S v|^{2} d x=\sigma_{1}^{-1} \mathbb{T}(r-\mu) \cdot(r-\mu)
$$

To see this we substitute $p=\chi_{2} \mu$ into (3.14) to get

$$
N\left(\chi_{2} \mu\right)=-\sigma_{1}^{-1} \sum_{\kappa \neq 0} e^{2 \pi i \kappa \cdot x} \frac{\kappa}{|\kappa|^{2}} \hat{\chi}_{2}(\kappa) \kappa \cdot \mu
$$


For our choice of $v$ we integrate by parts on the surface of each particle to find:

$$
\begin{aligned}
& \int_{\Gamma} e^{-2 \pi i \kappa \cdot y} T v d s=\sum_{i} \int_{\partial Y_{i}} e^{-2 \pi i \kappa \cdot y} T\left(r_{j} z^{i}\right) d s \\
& =-\sum_{i} \int_{\partial Y^{i}}\left(r_{i} z^{j}\right) \delta\left(e^{-2 \pi i \kappa \cdot y}\right) d s=(2 \pi i) \sum_{i} \int_{\partial Y^{i}} e^{-2 \pi i \kappa \cdot y} \kappa \cdot\left(\mathbf{n} \times h^{j}\right) r_{j} d s \\
& \quad=2 \pi i \sum_{i} \int_{Y^{i}} e^{-2 \pi i \kappa \cdot y} \kappa \cdot\left(e^{j}+(-2 \pi i) \kappa \times h^{j}\right) r_{j} d s .
\end{aligned}
$$

Noting that for $\kappa \neq 0, \hat{\chi}_{1}(\kappa)=-\hat{\chi}_{2}(\kappa)$, and $\kappa \cdot\left(\kappa \times h^{j}\right)=0$, we find that

$$
S v=\sigma_{1}^{-1} \sum_{\kappa \neq 0} e^{2 \pi i \kappa \cdot x} \frac{\kappa}{|\kappa|^{2}} \hat{\chi}_{2}(\kappa) \kappa \cdot r .
$$

Identity 6.6 follows, observing that the projection $\mathbb{P}$ is given by $\mathbb{P} p=\sum_{\kappa \neq 0} e^{2 \pi i \kappa \cdot x}\left(\kappa /|\kappa|^{2}\right) \hat{p}(\kappa)$. $\kappa$ and an application of Parcevals identity.

Carrying out the optimization implied by (6.1) we obtain the lower bound:

Theorem 6.2 For all $E$ in $\mathbb{R}^{3}$ one has,

$$
\sigma^{e} \boldsymbol{E} \cdot \boldsymbol{E} \geq \sigma_{1} \boldsymbol{E} \cdot \boldsymbol{E}+\left(\begin{array}{cc}
\theta_{2}\left(\sigma_{2}-\sigma_{1}\right)^{-1} I+\sigma_{1}^{-1} \mathbb{T} & -\sigma_{1}^{-1} \mathbb{T} \\
-\sigma_{1}^{-1} \mathbb{T} & \alpha^{-1} \mathbb{Z}+\sigma_{1}^{-1} \mathbb{T}
\end{array}\right)^{-1}\left(\begin{array}{c}
\theta_{2} \boldsymbol{E} \\
\theta_{1} \boldsymbol{E}
\end{array}\right) \cdot\left(\begin{array}{c}
\theta_{2} \boldsymbol{E} \\
\theta_{1} \boldsymbol{E}
\end{array}\right)
$$

For statistically isotropic composites $\mathbb{Z}=g_{3} I, \mathbb{T}=\frac{\sigma_{1} \sigma_{2}}{d} I$ where

$$
g_{3}=(1 / 3)\left(\sum_{i}\left(\int-\partial Y^{i}\left|y^{i}\right|^{2} d s-\int_{\partial Y^{i}}\left(\mathbf{n}_{1}^{2}\left(y_{3}^{i}\right)^{2}+\mathbf{n}_{2}^{2}\left(y_{1}^{i}\right)^{2}+\mathbf{n}_{3}^{2}\left(y_{2}^{i}\right)^{2}\right) d s\right)\right) .
$$

Since $|\mathbf{n}|^{2}=1$ it follows that $g_{3} \geq 0$.

We introduce the function defined for $t \geq 0$ given by

$$
L B_{3}(t)=\sigma_{1}+\frac{\theta_{2} t+\theta_{1}^{2} \beta+\theta_{1} /\left(3 \sigma_{1}\right)}{\left.\beta+\theta_{1} /\left(3 \sigma_{1}\right)\right) t+\theta_{1} \theta_{2} \beta /\left(3 \sigma_{1}\right)} .
$$

Here $\beta=\left(\sigma_{2}-\sigma_{1}\right)^{-1}$.

It is easily seen that this function is monotone decreasing in $t$. The lower bound on the effective conductivity for statistically isotropic composites is given by:

\section{Corollary 6.3}

$$
\sigma^{e} \geq L B_{3}\left(g_{3} / \alpha\right)
$$


This lower bound holds for all isotropic particulate composites.

For two-dimensional composites we make the same choice of bulk polarization (i.e., $p=\chi_{2} \mu, \mu$ in $\mathbb{R}^{3}$ ), however, on the $i^{\text {th }}$ particle we choose the surface polarization to be of the form $v=\left(r \cdot \mathcal{R} y^{i}\right) t$. Here, $t$ is the tangent vector in the plane perpendicular to the generators of the cylinders and $\mathcal{R}$ is a counter-clockwise rotation of $\pi / 2$ radians in the plane.

For this choice we obtain the lower bound on the in plane conductivity given by:

Corollary 6.4 Two-dimensional lower bound

$$
\sigma^{e} \geq L B_{2}\left(g_{2} / \alpha\right)
$$

where

$$
L B_{2}(t)=\sigma_{1}+\frac{\theta_{2} t+\theta_{1}^{2} \beta+\theta_{1} /\left(2 \sigma_{1}\right)}{\left(\beta+\theta_{1} /\left(2 \sigma_{1}\right)\right) t+\theta_{1} \theta_{2} \beta /\left(2 \sigma_{1}\right)}
$$

and

$$
g_{2}=(1 / 2)\left(\sum_{i} \int_{\partial Y^{i}}\left|y^{i}\right|^{2} d s\right) .
$$

If we know the shape of each inclusion one can adjust the surface polarization to give tighter bounds for three dimensional particulate composites. We suppose that the particulate suspension is an isotropic polydisperse suspension of spheres of radii $a_{1}, a_{2}, \ldots, a_{N}$. We observe that on the surface $\left|y^{i}\right|=a_{i}$, we have

$$
\Delta\left(r \cdot y^{i}\right)=\frac{-2}{a_{i}^{2}} r \cdot y^{i}=\frac{-2}{a_{i}} r \cdot n
$$

for any $r$ in $\mathbb{R}^{3}$.

Motivated by (6.17) we choose the surface polarization on the $i^{\text {th }}$ sphere to be of the form $v=a^{i}\left(\delta\left(r \cdot y^{i}\right)\right.$ and so $T v=\frac{a^{i}}{2} \Delta\left(r \cdot y^{i}\right)=-r \cdot n$.

Thus

$$
\sum_{i} \int_{\partial Y_{i}} e^{-2 \pi i \kappa \cdot y} T v d s=(2 \pi i) \kappa \cdot r \hat{\chi}_{1}(\kappa)
$$

and the identity (6.6) holds. Evaluation of all integrals in the variational principle and optimization gives:

Corollary 6.5 Three-dimensional lower bound for isotropic polydisperse suspensions of spheres.

$$
\sigma^{e} \geq L B_{3}(m / \alpha)
$$

where

$$
m=\frac{\theta_{1}}{2}\langle a\rangle
$$


We apply the general lower bound given in Corollary 6.3 to the case of polydisperse suspensions of spheres. We find that for this geometry $g_{3}=\frac{4}{5} \theta_{1}\langle a\rangle$. From the monotonicity of the function $L B_{3}(t)$ and the inequality $m<g_{3}$ it follows that

$$
L B_{3}(m / \alpha)=L B_{3}\left(\frac{\theta_{1}}{2}\langle a\rangle / \alpha\right)>L B_{3}\left(\frac{4}{5} \theta_{1}\langle a\rangle / \alpha\right) .
$$

Thus the bound given by Corollary 6.5 is a strict improvement over the bound given by Corollary 6.3 for polydisperse suspension of spheres.

In two dimensions one observes that the class of trials used in the bound (6.14) includes the scaled tangential gradients of affine functions and so no special treatment is needed, i.e., for circles $v=\left(r \cdot R y^{i}\right) t=a^{i} \delta\left(r \cdot y^{i}\right)$.

The two-dimensional bound (6.14) was obtained earlier in Lipton (1995) through the use of reciprocal relations and bounds on the effective conductivity with interfacial contact resistance given in Lipton and Vernescu (1996).

We have observed that the bounds $L B_{2}(t)$ and $L B_{3}(t)$ are monotonic decreasing. From this we see for $\alpha=0$ that $g_{3} / \alpha=\infty$ and

$$
\sigma^{e} \geq L B_{3}\left(g_{3} / \alpha\right) \geq L B(\infty)=H S_{-} .
$$

Here $H S_{-}$is the Hashin Shtrikman (1962) lower bound for isotropic perfectly bonded twophase composites.

\section{Size Effects, Inverse Problems, and Energy Minimizing Polydisperse Suspensions of Spheres}

We consider the behaviour of the upper bound in the limit of infinite interfacial surface area. We fix volume fractions and pass to the infinite interfacial surface area limit, (i.e., $s \rightarrow \infty)$ to find that the upper bound behaves like $\sigma_{2} \stackrel{*}{\sigma}_{\infty}+O\left(s^{-1}\right)$. Here $\sigma_{2} \tilde{\sigma}_{\infty}$ is the effective conductivity associated with the same microgeometry but with perfectly conducting particles in a matrix of conductivity $\sigma_{2}$.

This behavior is physically consistent with the behavior of effective tensors under a rescaling of the geometry. It is shown in Lipton (1995), that the effective conductivity of a $k^{-1}$ periodic composite is identical to that of an unrescaled composite, but with tangential conductivity increased by the factor $k$, see Theorem 4.2 of Lipton (1995). Thus as $k$ tends to infinity so does the interfacial surface area and the effective conductivity of the $k^{-1}$ periodic composite tends to that of the unrescaled composite with infinite tangential conductivity. The effective conductivity for such a composite is given precisely by $\sigma_{2} \stackrel{*}{\sigma}_{\infty}$. Thus we see that the upper bound captures the asymptotics consistent with effect tensors under rescaling. Indeed we see that its optimal in this limit. 
Next we consider three dimensional isotropic particulate suspensions. As before we take the particle conductivity to be $\sigma_{1}$ and that of the matrix to be $\sigma_{2}$. We restrict discussion to three-dimensional composites as the related two-dimensional problem was worked out earlier in Lipton (1995). For fixed component volume fractions we use the monotonicity of the bounds in the interfacial parameters $g_{3}, m$, and specific surface area $s$ to exhibit new size effects. The bounds will serve as a tool for understanding the role of surface energy in problems of energy minimizing arrangement of polydisperse suspensions of spheres. We recall the parameter $P_{c r}^{3}=2 \alpha /\left(\sigma_{2}-\sigma_{1}\right)$ and start by considering monodisperse suspensions of spheres and state the following:

Theorem 7.1 Given that the common radius of a monodisperse suspension of spheres is $a$, then

$$
\begin{array}{lll}
\sigma^{e}>\sigma_{2} & \text { for } & a<P_{c r}^{3}, \\
\sigma^{3}<\sigma_{2} & \text { for } & a>P_{c r}^{3},
\end{array}
$$

and

$$
\sigma^{e}=\sigma_{2} \quad \text { for } \quad a=P_{c r}^{3} .
$$

Proof: For monodisperse suspensions of common radius $a$ the geometric parameters $m$ and $s$ are given by

$$
m=\theta_{1} a / 2, \quad s=3 \theta_{1} a^{-1} .
$$

When $a=P_{c r}^{3}$ substitution of (7.4) into the upper and lower bounds (5.10) and (6.19) gives

$$
\sigma_{2}=U B=L B_{3}
$$

and (7.3) follows. Inequalities (7.1) and (7.2) follow from the monotonicity of the bounds in the geometric parameters.

Theorem 7.1 shows that the effective conductivity increases at fixed volume fraction as the sphere diameter decreases. For polydisperse suspensions of spheres we state the following:

Theorem 7.2 Size effects for polydisperse suspensions of spheres.

For particle volume fraction $\theta_{1}$ fixed:

$$
\begin{cases}\text { If }\langle a\rangle<P_{c r}^{3} & \text { then } \sigma^{e} \geq \sigma_{2}, \text { and } \\ \text { if }\left\langle a^{-1}\right\rangle^{-1}>P_{c r}^{3} & \text { then } \sigma^{e} \leq \sigma_{2} .\end{cases}
$$

Proof: For polydisperse suspensions, the geometric parameter $m=\theta_{1}\langle a\rangle / 2$ and the lower bound (6.19) is strictly monotonically increasing as the mean radius tends to zero. For $\langle a\rangle=P_{c r}^{3}$ one has $L B_{3}(m / \alpha)=\sigma_{2}$, and the first statement follows. The second statement 
follows from (5.12), noting that $U B$ is increasing in $\left\langle a^{-1}\right\rangle$ together with (5.14). We note that identical results hold for two-dimensional composites.

We now consider particulate suspensions with no assumption on particle shape or distribution other than that the resulting effective conductivity is isotropic. For this case we have the:

Theorem 7.3 for fixed particle fraction $\theta_{1}$, if the specific surface area is bounded above by $\theta_{1}\left(P_{c r}^{3}\right)^{-1}$, then

$$
\sigma^{e} \leq \sigma_{2}
$$

Proof: For $s=3 \theta_{1}\left(P_{c r}^{3}\right)^{-1}$ it follows from (5.14) that the upper bound $U B=\sigma_{2}$. Moreover, since the upper bound is monotone increasing in $s$ one has that $U B \leq \sigma_{2}$ for $s \leq 3 \theta_{1}\left(P_{c r}^{3}\right)^{-1}$ and the theorem follows.

From its definition, its evident that the effective conductivity is equivalent to the energy dissipated inside the two-phase conductor, see equation 2.7. In this regard, we see that Theorems 7.1, 7.2, and 7.3 are energy dissipation theorems for a system with bulk and interfacial energy. In what follows we consider only isotropic polydisperse suspensions of spheres. We fix the volume fraction of spheres and examine the the role of surface energy in selecting a suspension with minimum isotropic effective conductivity. In what follows we present a necessary condition on the size distribution of the spheres appearing in the optimal suspension. We remark that it is not known if a minimal suspension exists. Instead, as with many problems, there may be no minimum but only a minimizing sequence of suspensions. For this case, the same necessary condition would hold in the appropriate sense for minimizing sequences of suspensions.

To fix ideas we suppose that the volume fraction of spheres satisfies the inequality:

$$
\theta_{1}<\pi / 6
$$

That is, we require the volume fraction to be less than a sphere of radius $1 / 2$ inscribed within the unit cell. We restrict the parameters $\sigma_{1}, \sigma_{2}, \alpha$ so that $P_{c r}^{3}$ satisfies, $\frac{4}{3} \pi\left(P_{c r}^{3}\right)^{3}<\theta_{1}$. This directs the discussion to cases where the volume of a single sphere of critical radius is strictly less than the volume fraction occupied by the suspension. We have the following theorem characterizing the optimal polydisperse suspension dissipating the least energy:

\section{Theorem 7.4 Optimal design necessary condition.}

Given $\theta_{1}, \sigma_{1}, \sigma_{2}, \alpha$ satisfying the constraints stated above, then the mean radius of the distribution of spheres dissipating the least energy is greater than $P_{c r}^{3}$.

Proof: From Theorem 7.2 we know if the mean radius lies below $P_{c r}^{3}$ then $\sigma^{e} \geq \sigma_{2}$. So to establish the theorem we construct a polydisperse suspension of spheres with mean radius greater than $P_{c r}^{3}$ with effective conductivity less than $\sigma_{2}$. 
The construction is easy in light of the constraints on $\theta_{1}, \sigma_{2}, \sigma_{2}$, and $\alpha$. We choose a suspension consisting of a single sphere centered in the period cell of radius " $a$ " such that $\frac{4}{3} \pi a^{3}=\theta_{1}$. Then for this suspension $s / 3 \theta_{1}=a^{-1}$. From the constraints we see that $a^{-1}<\left(P_{c r}^{3}\right)^{-1}$, therefore $s<3 \theta_{1}\left(P_{c r}^{3}\right)^{-1}$ and so from Theorem 7.3 we have $\sigma^{e}<\sigma_{2}$ and the theorem is proved.

This theorem shows that the scale of the heterogeneity plays a role in the extremal energy dissipation for an isotropic polydisperse suspension of spheres. This is in contrast to optimal layout problems with perfect transmission between phases where scale plays no role in the optimal design, see Lurie and Cherkaev (1986) and Murat and Tartar (1985).

\section{Inverse Problems}

We show how to use the monotonicity of the bounds in the geometric parameters to characterize the size distribution of a polydisperse suspension of spheres from measured values of the effective conductivity.

Assuming that the volume fractions $\theta_{1}, \theta_{2}$ one known as well as the values $\sigma_{1}, \sigma_{2}, \alpha$ we will bound the averages $\langle a\rangle$ and $\left\langle a^{-1}\right\rangle^{-1}$ in terms of the measured value of $\sigma^{e}$. Indeed we have the:

Theorem 8.1 Characterization of the size distribution for suspensions of spheres.

If $\sigma^{e}\left\langle\sigma_{2}\right.$ then $\langle a\rangle>P_{c r}^{3}$, and if $\sigma^{e}>\sigma_{2}$ then $\left\langle a^{-1}\right\rangle^{-1}<P_{c r}^{3}$.

We note that this follows immediately from Theorem 7.2.

\section{Concluding Remarks}

The variational principles given in Section 3 can be extended to the multiphase case by choosing the comparison conductivity $\gamma$ to render the difference between the local conductivity and the comparison conductivity, definite. The use of a comparison conductivity is standard for perfectly bonded multiphase composites and was introduced in the celebrated work of Hashin and Shtrikman (1962).

\section{A Appendix}

We show the tensor $\sigma_{\infty}$ defined by (3.10) is invertible and symmetric. Noting that $\int_{\partial Q}\left(\nabla \psi^{\mathrm{c}}+\right.$ $\mathbf{c}) \cdot \mathbf{n} \psi^{\mathbf{c}} d s=0$, applying (3.1) and (3.2) and integrating by parts gives

$$
\sigma_{\infty} \mathbf{c} \cdot \mathbf{c}=\int_{Q}\left|\nabla \psi^{\mathbf{c}}+\mathbf{c}\right|^{2} d x,
$$


and one easily verifies that

$$
\sigma_{\infty} \mathbf{c} \cdot \mathbf{c}=\min _{\substack{\psi \in V \\ \psi=-\mathbf{c} x}} \int_{\text {on } \Gamma}|\nabla \psi+\mathbf{c}|^{2} d x .
$$

It follows from (A.2) that $\sigma_{\infty}$ is symmetric. For particulate composites $\psi^{\mathbf{c}}=-\mathbf{c} \cdot x$ inside the particles and $\sigma_{\infty}=\dot{\sigma}_{\infty}$, where $\tilde{\sigma}_{\infty}$ is the effective conductivity for a suspension of perfectly conducting inclusions. Taking the Frenchel dual of the integrand and an exchange of minimization and maximization gives

$$
\sigma_{\infty} \mathbf{c} \cdot \mathbf{c} \geq \max _{p \in L^{2}(Q)^{d}}\left[2 \int_{\partial Q}(p \cdot \mathbf{n})(\mathbf{c} \cdot x) d s-\int_{Q}|p|^{2} d x\right] .
$$

Where $\operatorname{div} p=0$ and $p \cdot \mathbf{n}$ is antiperiodic.

For the choice $p=t \mathbf{c}$ where $t$ is a scalar we obtain $\sigma_{\infty} \mathbf{c} \cdot \mathbf{c} \geq|\mathbf{c}|^{2}\left(2 t-t^{2}\right)$. Optimization over $t$ gives

$$
\sigma_{\infty} \mathbf{c} \cdot \mathbf{c} \geq|\mathbf{c}|^{2}
$$

and invertibility of $\sigma_{\infty}$ follows.

\section{References}

[1] S. Chandrasekhar (1965) The stability of a rotating liquid drop, Proc. Roy. Soc. Lond. (A), 286, pp. 1-26.

[2] I. Ekeland and R. Temam (1976) Convex Analysis and Variational Problems, NorthHolland, Amsterdam.

[3] Z. Hashin and S. Shtrikman (1962) A variational approach to the theory of effective magnetic permeability of multiphase materials, J. Appl. Phys., 33, pp. 3125-3131.

[4] S. Kobayashi and K. Nomizu (1969) Foundations of Differential Geometry, Vol. 2, Interscience, New York .

[5] R.V. Kohn and G.W. Milton (1988) Variational bounds on the effective moduli of anisotropic composites, J. Mech. Phys. Solids, 36, pp. 597-629.

[6] R. Lipton (1995) Reciprocal relations, bounds, and size effects for composites with highly conducting interface, IMA Preprint 1339; to Appear in SIAM J. Appl. Math.

[7] R. Lipton and B. Vernescu (1996) Composites with imperfect interface, Proc. Roy. Soc. Lond. (A), 452, pp. 329-358. 
[8] R. Lipton and B. Vernescu (1995) Variational methods, size effects and extremal microgeometries for elastic composites with imperfect interface, Math. Models Methods Appl. Sci., 8, pp. 1139-1173.

[9] K.A. Lurie and A.V. Cherkaev (1986) Effective characteristics of composite materials and the optimal design of structural elements, Uspekhi Mekhaniki, Advances in Mechanics, 9.

[10] F. Murat and L. Tartar, (1985) Calcul des Variations et homogégéisation: Théorie et applications en physique, Coll. de la Dir. des Etudes et Reserches de Electr. del France, Eyrolles, Paris, pp. 319-370.

[11] H. Pham Huy and E. Sanchez-Palencia (1974) Phénomènes de à traverse des coucheo minces de conductivité élevée, Journal of Mathematical Analysis and Applications, 47, pp. 284-309.

[12] S. Torquato and M.D. Rintoul (1995) Effect of the interface on the properties of composite media, Phys. Rev. Letters, 75, pp. 4067-4070. 